\title{
Mössbauer Investigations and Photoemission Studies of the Fe $3 s$ Spin Splitting in Some Fe-Ni Alloys
}

\author{
M. Ka̧dziołka-Gawę, W. Zarek, E. Talik and E. Popiel \\ Institute of Physics, University of Silesia \\ Uniwersytecka 4, 40-007 Katowice, Poland
}

\begin{abstract}
The magnetic properties, crystal and electronic structure for $\mathrm{Fe}_{1-x} \mathrm{Ni}_{x}$ $(x=0.30,0.325,0.375)$ alloys and austenitic steel were studied using magnetostatic, Mössbauer effect methods, X-ray diffraction and X-ray photoelectron spectroscopy. The compositions of the investigated $\mathrm{Fe}-\mathrm{Ni}$ alloys were chosen like that to exist on left, right and in inside of invar range. Two different magnetic moments (low and high) of Fe atom were observed. Measurement results do not show antiferromagnetic order in $\mathrm{Fe}-\mathrm{Ni}$ alloys.
\end{abstract}

PACS numbers: 82.80.Ej, 82.80.Pv

\section{Introduction}

The physical properties of $\mathrm{Fe}-\mathrm{Ni}$ alloys and connected with them invar effects were subject of great number of theoretical and experimental works. The number of models have been suggested to explain invar effect, i.e.: "two-gamma state" model [1], latent antiferromagnetism [2], weak itinerant ferromagnetism, mixed exchange model [3]. At present, the most popular models are two-gamma state model and mixed exchange model [4-7]. The first of them postulates that Fe atom can exist in two electronic states: low-spin state with low volume and antiferromagnetic order and high-spin with high volume ferromagnetic state. The mixed exchange model postulates the existence of localized magnetic moments of $\mathrm{Fe}$ and $\mathrm{Ni}$ atoms and mixed exchange interaction between them: $J_{\mathrm{FeNi}}>0$, $J_{\mathrm{NiNi}}>0, J_{\mathrm{FeFe}}<0$. The magnetic moment of $\mathrm{Fe}$ atom depends on local environment and can order parallel or anti-parallel with respect to the net magnetization. The Mössbauer measurements performed by monochromatic circularly polarized source [8] showed that the average cosine between local iron magnetic moment and the average magnetization in the invar $\mathrm{Fe}_{0.65} \mathrm{Ni}_{0.35}$ is the same in whole sample.

Some useful information about physical properties of $\mathrm{Fe}-\mathrm{Ni}$ alloys can be obtained from investigation of their electronic structure. Some authors $[9,10]$ supposed that the multiplet splitting of the Fe $3 s$ core level can be used diag- 
nostically to identify the presence of local magnetic moment on the Fe site. The changes of the multiplet splitting parameters of the $3 s$ spectra of $d$ metals give the information about the tendencies of the changes in the local magnetic moment and the localization of the $3 d$ electrons. However, correlation between magnetic moment and $3 s$ multiplet splitting is not unequivocal, yet [11]. Until now, not many experimental works concerning X-ray photoelectron spectroscopy (XPS) studied for $\mathrm{Fe}-\mathrm{Ni}$ alloys were made. The investigations $[12,13]$ obtained from a study of the multiplet splitting of the $\mathrm{Fe} 3 s$ core level of $\mathrm{Fe}_{0.65} \mathrm{Ni}_{0.35}$ showed existence of Fe atom in two different states: low-spin and high-spin state.

In this paper we present the results of crystal structure, magnetic, Mössbauer effect and XPS measurements of $\mathrm{Fe}_{1-x} \mathrm{Ni}_{x}(x=0.3,0.325,0.375)$ alloys.

\section{Experimental}

The polycrystalline $\mathrm{Fe}_{1-x} \mathrm{Ni}_{x}(x=0.30,0.325,0.375)$ alloys were prepared by the arc-melting using stoichiometric quantities of the starting metals in an argon atmosphere. Ingots were several times re-melted to ensure homogeneity. X-ray diffraction studies were performed at room temperature on powdered samples using a Siemens D5000 X-ray diffractometer and $\mathrm{Cu} K_{\alpha}$ radiation.

The magnetic measurement of the investigated samples were performed at temperatures up to $600 \mathrm{~K}$ in the magnetic fields up to $1.2 \mathrm{~T}$ using the Faraday method.

The $\mathrm{Fe}^{57}$ Mössbauer spectra were recorded at room temperature using a constant acceleration spectrometer with $\mathrm{Co}^{57}: \mathrm{Pd}$ source. Metallic iron powder and sodium nitroprusside powder were used for velocity calibrations of the Mössbauer spectrometer. The obtained spectra were fitted as a superposition of several Zeeman sextets, by means of hyperfine field distribution and by the fluctuating hyperfine field method [14]. The discrete analysis, local-environment effect [8] have been applied to analysis the Mössbauer spectra of investigated compounds.

The XPS spectra of the investigated $\mathrm{Fe}_{1-x} \mathrm{Ni}_{x}(x=0.30,0.325,0.375)$ alloys, austenitic steel and pure $\mathrm{Fe}$ and Ni were performed with monochromatized Al $K_{\alpha}$ radiation at room temperature using PHI 5700/660 Physical Electronics spectrometer. The samples were cleaned in situ by argon bombardment. The data of the Fe $3 s$ spectra were analyzed by fitting two Doniach-Sunjic (DS) [15] line shapes.

\section{Results and discussion}

The X-ray diffraction measurements show that the alloy with $x=0.30$ has single body-centered cubic (bcc) phase, alloy with $x=0.375$ and austenitic steel have single face-centered cubic (fcc) phase and alloy with $x=0.325$ contains both these phases. Detailed analysis of diffraction spectra showed atomic disorder of the crystal structure. Results of crystallographic and magnetic measurements are presented in Table I. 
TABLE I

Crystal structure, lattice parameter $a$, magnetic moment $\mu$ at room temperature and the Curie temperature $T_{\mathrm{C}}$ of $\mathrm{Fe}_{1-x} \mathrm{Ni}_{x}$ alloys.

\begin{tabular}{c|c|c|c|c}
\hline \hline$x$ & Crystal structure & $a[\AA]$ & $T_{\mathrm{C}}[\mathrm{K}]$ & $\mu\left[\mu_{\mathrm{B}} /\right.$ f.u. $]$ \\
\hline 0.30 & bcc & 2.858 & $>650$ & 2.14 \\
\hline 0.325 & bcc & 2.861 & $>650$ & 1.67 \\
& fcc & 3.582 & 382 & \\
\hline 0.375 & fcc & 3.587 & 417 & 1.57
\end{tabular}

Magnetic studies show that the investigated $\mathrm{Fe}-\mathrm{Ni}$ alloys are ferromagnetic with the Curie temperature above room temperature and the austenitic steel is paramagnetic at room temperature.

The Mössbauer absorption spectra, diagrams of hyperfine field (HF) distributions and distribution of probabilities $P(n)$ that the Fe atom has $n$ Fe atoms as nearest neighbors for investigated compounds are presented in Fig. 1.

The hyperfine field distribution for $\mathrm{Fe}_{0.70} \mathrm{Ni}_{0.30}$ is narrow and connected with different local configurations of the atom in atomically disordered lattice. The shape of the Mössbauer spectrum for this alloy can be explained by two sextets and a single line. The hyperfine field distributions for $\mathrm{Fe}_{0.675} \mathrm{Ni}_{0.325}$ and $\mathrm{Fe}_{0.625} \mathrm{Ni}_{0.375}$ alloys are broad and two regions can be distinguished: one with high value of magnetic moments and second with low or zero value. The Mössbauer spectra of the alloy with $x=0.375$ was fitted by four sextets and two single lines and for alloy with $x=0.325$ by three broad sextets and also by two single lines. The fitting parameters of the Mössbauer spectra are presented in Table II. The austenite steel is paramagnet at room temperature and the Mössbauer spectrum of this compound shows only single line with isomer shift equal to $-0.11 \mathrm{~mm} / \mathrm{s}$.

We assume that the fields connected with sextets are effect of surrounding $\mathrm{Fe}$ atom by zero to nine and Fe atoms in first coordination sphere. It confirms with theoretical and experimental works $[16,17]$. The single lines are probably connected with Fe atoms surrounded by ten to twelve Fe atoms $(\gamma-\mathrm{Fe})$. These two different magnetic field regions can be connected with two different magnetic moments in fcc $\mathrm{Fe}-\mathrm{Ni}$ alloys.

The spectra of fcc Fe-Ni alloys and austenite steel also were analyzed using the fluctuating hyperfine fields method [18]. The spectra were fitted using from one to four fluctuating hyperfine fields $H_{\mathrm{hf}}$. We obtained good compatibility with experiment. The parameters from the analysis are in Table III.

Rancourt et al. [19] observed in $\mathrm{Fe}_{0.65} \mathrm{Ni}_{0.35}$ three crystallographic and magnetic different phases. The first is high-spin phase connected with face-centered cubic lattice $(\gamma$-phase) which is ferromagnetic below $500 \mathrm{~K}$ with magnetic moment $\mu_{\mathrm{Fe}} \approx 2.8 \mu_{\mathrm{B}}$, the second high-spin $\left(\mu_{\mathrm{Fe}} \approx 2.2 \mu_{\mathrm{B}}\right)$ phase is connected with 

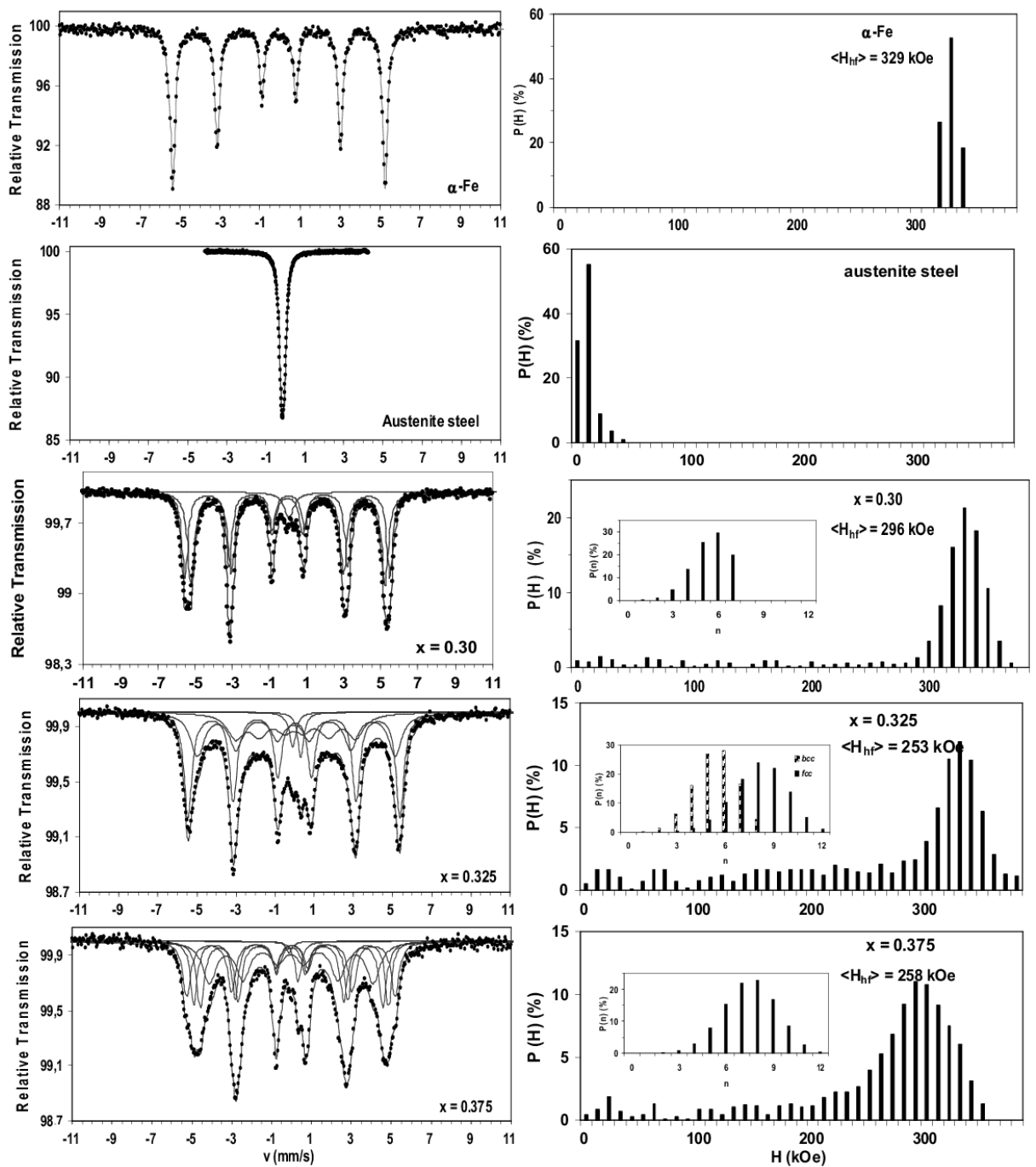

Fig. 1. The Mössbauer spectra and respective hyperfine field (HF) distribution and distribution of probability $P(n)$ that Fe atom has $n$ Fe atoms as nearest neighbors for the investigated compounds at room temperature.

body-centered cubic phase ( $\alpha$-phase) and is ferromagnetic below $1000 \mathrm{~K}$, the third low-spin $\left(\mu_{\mathrm{Fe}} \approx 0.5 \mu_{\mathrm{B}}\right)$ phase with fcc structure $\left(\gamma^{\prime}\right.$-phase) is antiferromagnetic below $20 \mathrm{~K}$. The $a$ and $\gamma^{\prime}$ phases appear as large precipitates. According to the Rancourt precipitates of the phase $\gamma^{\prime}$ cause domain wall pinning and lead to formation of reentrant spin glass. The investigation made by Satuła et al. [8] did not show antiferromagnetic component in $\mathrm{Fe}-\mathrm{Ni}$. In fcc $\mathrm{Fe}_{0.625} \mathrm{Ni}_{0.375}$ sample we can distinguish three different hyperfine fields (HF): 300, 260 and $20 \mathrm{kOe}$ (Fig. 1) 
TABLE II

The fitting parameters of the Mössbauer spectra: hyperfine magnetic field $H_{\mathrm{hf}}$, isomer shift IS and contribution of components in spectrum for investigated compounds.

\begin{tabular}{c|c|c|c}
\hline \hline Sample & $H_{\text {hf }}[\mathrm{kOe}]$ & IS [mm/s] & Contribution [\%] \\
\hline$\alpha-\mathrm{Fe}$ & 330 & 0.000 & 100 \\
\hline $\mathrm{Fe}_{0.70} \mathrm{Ni}_{0.30}$ & 324 & 0.051 & 48.43 \\
& 342 & 0.057 & 47.01 \\
\hline & 191 & 0.050 & 20.80 \\
$\mathrm{Fe}_{0.675} \mathrm{Ni}_{0.325}$ & 315 & 0.055 & 30.02 \\
& 337 & 0.042 & 43.87 \\
\hline & 256 & 0.009 & 27.36 \\
$\mathrm{Fe}_{0.625} \mathrm{Ni}_{0.375}$ & 285 & 0.034 & 25.20 \\
& 303 & 0.035 & 21.22 \\
& 325 & 0.041 & 22.54
\end{tabular}

TABLE III

The parameters from the analysis by the fluctuating hyperfine fields method: hyperfine magnetic field $H_{\mathrm{hf}}$ and relaxation time $\tau$, obtained for fcc $\mathrm{Fe}_{1-x} \mathrm{Ni}_{x}$ alloys and austenite.

\begin{tabular}{c|c|c}
\hline \hline Sample & $H_{\mathrm{hf}}[\mathrm{kOe}]$ & $\tau[\mathrm{s}]$ \\
\hline \multirow{3}{*}{0.325} & 348 & $2.0 \times 10^{-8}$ \\
& 326 & $1.1 \times 10^{-7}$ \\
& 207 & $8.3 \times 10^{-8}$ \\
& 10 & $1.1 \times 10^{-12}$ \\
\hline \multirow{3}{*}{0.375} & 337 & $8.8 \times 10^{-9}$ \\
& 210 & $1.1 \times 10^{-8}$ \\
& 12 & $2.3 \times 10^{-12}$ \\
\hline Austenite steel & 8 & $7.1 \times 10^{-12}$
\end{tabular}

which are connected with various local environments of Fe atoms which yields high and low magnetic moment. These fields are distinctly visible in the diagram of hyperfine fields distribution of the two-phase $\mathrm{Fe}_{0.675} \mathrm{Ni}_{0.325}$.

We suppose that low magnetic moment of the Fe atom $(\mathrm{HF} \approx 20 \mathrm{kOe})$ is connected with Fe atoms surrounded by $10 \div 12$ other Fe atoms, like in $\gamma$-Fe. The presence of single lines was observed also in the Mössbauer spectra of alloys with $x=0.375$ and $x=0.325$ at low temperature $\approx 20 \mathrm{~K}$ (Fig. 2). The contribution of these lines to the whole spectra is the same as at room temperature. On basis 

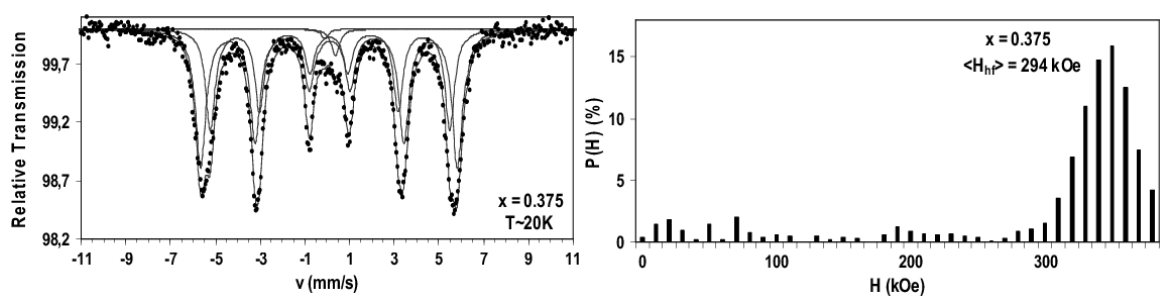

Fig. 2. The Mössbauer spectra and respective hyperfine field (HF) distribution for $\mathrm{Fe}_{0.625} \mathrm{Ni}_{0.375}$ at temperature $\approx 20 \mathrm{~K}$.

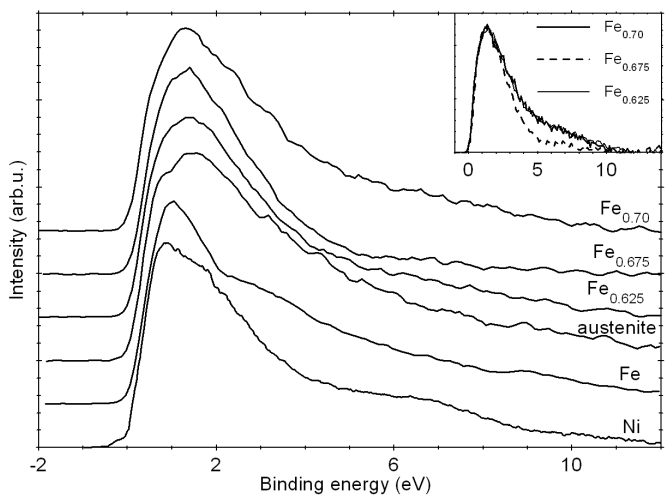

Fig. 3. The valence band XPS spectra of $\alpha-\mathrm{Fe}, \mathrm{Ni}$, and investigated compounds.

of these observations we suppose that the Fe atoms with low magnetic moment remain paramagnetic in the whole temperature range.

The presence of the Fe atoms with high and low magnetic moment in the investigated samples confirms investigations of electronic structure by XPS method.

The valence band XPS spectra of investigated compounds are shown in Fig. 3. The structure of the valence bands of $\alpha$-Fe and $\mathrm{Ni}$ manifest themselves in the valence bands of investigated alloys, also some contribution of austenite valence band shape in alloys with $x=0.325$ and $x=0.375$ is evident. Figure 4 shows the photoelectron spectra of the Fe $3 s$ core level in pure $\alpha$-Fe, austenite $(\gamma$-Fe) and in the investigated alloys. In Table IV we list parameters of $3 s$ spin splitting for investigated compounds. The spectra of pure iron and $\mathrm{Fe}_{0.70} \mathrm{Ni}_{0.30}$ show the well-known doublet structure. This doublet is made up from a pair of exchange-split peaks.

The main peak has the minority spin character. For the austenite steel, $\mathrm{Fe}_{0.675} \mathrm{Ni}_{0.325}$ and $\mathrm{Fe}_{0.625} \mathrm{Ni}_{0.375}$ besides the main peak there are also two additional peaks. These three peaks can be interpreted as two-multiplet splitting with $\Delta E_{1} \approx$ $3.8 \mathrm{eV}$ and $\Delta E_{2} \approx 7 \mathrm{eV}$ which correspond with high and low spin state of the $\mathrm{Fe}$ atoms in the austenite and investigated alloys. The high spin state in our samples is probably as in $\alpha$-Fe (bcc). The low spin state can be connected with very low 


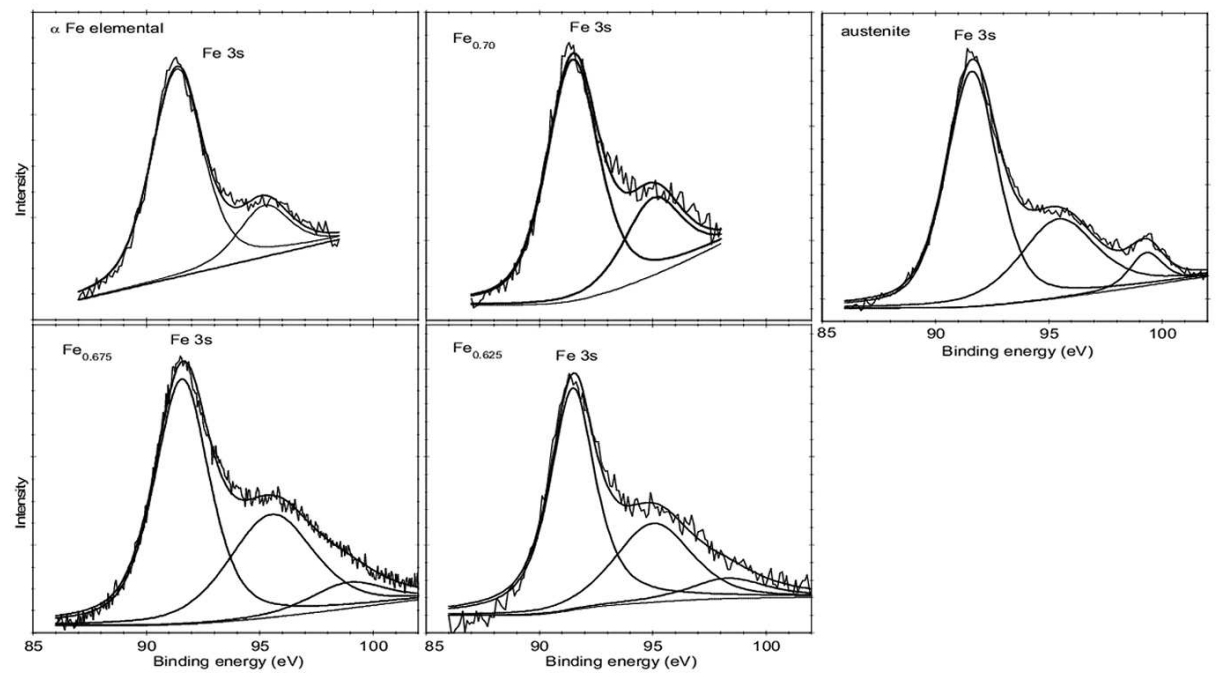

Fig. 4. The Fe $3 s$ spectra of the pure $\mathrm{Fe}$, austenitic steel and $\mathrm{Fe}_{1-x} \mathrm{Ni}_{x}(x=0.30$, $0.325,0.375)$ alloys, after subtraction of background.

\section{TABLE IV}

Fe $3 s$ XPS peak splitting $\left(\Delta\right.$, in eV), $I_{b} / I_{a}$ is the ratio of additional peak to main peak intensity, $2 \gamma$ is the Lorentzian broadening of the main Fe $3 \mathrm{~s}$ peak.

\begin{tabular}{c|c|c|c}
\hline \hline Sample & $\Delta E[\mathrm{eV}]$ & $2 \gamma[\mathrm{eV}]$ & $I_{b} / I_{a}$ \\
\hline $\mathrm{Fe}$ & 3.84 & 2.70 & 0.25 \\
\hline Austenite steel & 3.90 & 2.70 & 0.40 \\
& 7.90 & & 0.30 \\
\hline $\mathrm{Fe}_{0.70} \mathrm{Ni}_{0.30}$ & 3.84 & 2.70 & 0.35 \\
\hline $\mathrm{Fe}_{0.675} \mathrm{Ni}_{0.325}$ & 3.94 & 2.88 & 0.62 \\
& 7.34 & & 0.15 \\
\hline $\mathrm{Fe}_{0.625} \mathrm{Ni}_{0.375}$ & 3.54 & 2.79 & 0.49 \\
& 6.84 & & 0.20
\end{tabular}

or zero magnetic moments. From our measurements results we cannot say what value exactly this magnetic moment has. The broadening of main $3 s$ peak $(2 \gamma)$ is comparable to that given elsewhere [11], the other components of the Fe $3 \mathrm{~s}$ core level spectra are usually $\approx 10 \div 60 \%$ broader which can arise from atomic disorder of crystal structure. 


\section{Summary}

From the above results we may conclude the following.

In our opinion two magnetic moments of the Fe atoms exist in fcc structure of $\mathrm{Fe}-\mathrm{Ni}$ alloys in invar regions. These different magnetic moments are results of local environment. The Fe atom has high moment when it is surrounded from zero to about nine Fe atoms. The low or zero magnetic moments appear when $\mathrm{Fe}$ atom has from about ten to eleven Fe atoms as the nearest neighbors. This low magnetic moment appears in fcc structure of $\mathrm{Fe}-\mathrm{Ni}$ alloys in invar composition range because only in this concentration range the probability of finding of the $\mathrm{Fe}$ atom surrounding by twelve other Fe atoms is different from zero.

The XPS spectra of Fe $3 s$ core level splitting confirm the presence of the $\mathrm{Fe}$ atom in high and low spin state. We suppose that the Fe atoms in low spin state remain paramagnetic even at the low temperature.

\section{References}

[1] R.J. Weiss, Proc. Phys. Soc. 82, 281 (1963).

[2] E.J. Kondorsky, V.L. Sedov, J. Appl. Phys. 31, 3315 (1979).

[3] E.P. Wohlfarth, J. Magn. Magn. Mater. 10, 120 (1979).

[4] S. Kamura, G. Lippmann, W. Schmatz, J. Magn. Magn. Mater. 5, 123 (1977).

[5] S.F. Dubinin, S.K. Sidorov, E.Z. Valiev, Phys. Status Solidi 46, 337 (1971).

[6] A.Z. Menshikov, J. Magn. Magn. Mater. 10, 205 (1979).

[7] J.B. Muller, J. Hesse, Z. Phys. B, Condens. Matter 54, 35 (1983).

[8] D. Satuła, K. Szymański, L. Dobrzyński, K. Rećko, J. Waliszewski, Nukleonika 48, 72 (2003).

[9] C. Binns, C. Norris, G.P. Williams, M.G. Barthes, H.A. Padmore, Phys. Rev. B 34, 8221 (1986).

[10] S.L. Qiu, R.G. Jordan, A.M. Begley, X. Wang, Y. Liu, Phys. Rev. B 46, 13004 (1992).

[11] J.F. van Acker, Z.M. Stadnik, J.C. Fuggle, H.J.W.M. Hoeslra, K.H.J. Buschow, G. Stroink, Phys. Rev. B 37, 6827 (1988).

[12] J.N. Shabanova, N.V. Keller, V.A. Sosnov, A.Z. Menshikov, J. Electron Spectrosc. Relat. Phenom. 114-116, 581 (2001).

[13] N.V. Lomova, J.N. Shabanova, A.G. Chirkov, A.G. Ponomaryov, J. Electron Spectrosc. Relat. Phenom. 156-158, 401 (2007).

[14] D.G. Rancourt, S.R. Julian, J.M. Daniels, J. Magn. Magn. Mater. 51, 83 (1985).

[15] S. Doniach, M. Sunjic, J. Phys. C 3, 285 (1970).

[16] N. Hamada, J. Phys. Soc. Japan 46, 1759 (1979).

[17] S. Mishra, P.A. Back, S. Foner, J. Phys. Chem. Solids 32, 1977 (1970).

[18] M. Blume, J.A. Tjon, Phys. Rev. 164, 446 (1967).

[19] D.G. Rancourt, H.H.A. Smit, R.C. Thiel, J. Magn. Magn. Mater. 66, 121 (1987). 